\title{
Improved switching using Fano resonances in photonic crystal structures
}

\author{
Heuck, Mikkel; Kristensen, Philip Trøst; Elesin, Yuriy; Mørk, Jesper
}

Published in:

Optics Letters

Link to article, DOI:

10.1364/OL.38.002466

Publication date:

2013

Document Version

Publisher's PDF, also known as Version of record

Link back to DTU Orbit

Citation (APA):

Heuck, M., Kristensen, P. T., Elesin, Y., \& Mørk, J. (2013). Improved switching using Fano resonances in photonic crystal structures. Optics Letters, 38(14), 2466-2468. https://doi.org/10.1364/OL.38.002466

\section{General rights}

Copyright and moral rights for the publications made accessible in the public portal are retained by the authors and/or other copyright owners and it is a condition of accessing publications that users recognise and abide by the legal requirements associated with these rights.

- Users may download and print one copy of any publication from the public portal for the purpose of private study or research.

- You may not further distribute the material or use it for any profit-making activity or commercial gain

- You may freely distribute the URL identifying the publication in the public portal

If you believe that this document breaches copyright please contact us providing details, and we will remove access to the work immediately and investigate your claim. 


\title{
Improved switching using Fano resonances in photonic crystal structures
}

\author{
Mikkel Heuck, ${ }^{1, *}$ Philip Trøst Kristensen, ${ }^{1}$ Yuriy Elesin, ${ }^{2}$ and Jesper Mørk $^{1}$ \\ ${ }^{1}$ Department of Photonics Engineering, Technical University of Denmark, Ørsteds Plads, Bygn. 343, \\ Lyngby 2800 Kgs., Denmark \\ ${ }^{2}$ Department of Mechanical Engineering, Technical University of Denmark, Nils Koppels Allé, Bygn. 403, \\ Lyngby 2800 Kgs., Denmark \\ *Corresponding author: mheu@fotonik.dtu.dk
}

Received March 22, 2013; revised May 21, 2013; accepted May 22, 2013; posted May 23, 2013 (Doc. ID 187650); published July 9, 2013

\begin{abstract}
We present a simple and robust structure for realizing asymmetric Fano transmission characteristics in photonic crystal waveguide-cavity structures. The use of Fano resonances for optical switching is analyzed using temporal coupled mode theory in combination with three-dimensional finite difference time domain simulations taking into account the signal bandwidth. The results suggest a significant energy reduction by employing Fano resonances compared to more well established Lorentzian resonance structures. A specific example of a Kerr nonlinearity shows an order of magnitude energy reduction. (C) 2013 Optical Society of America
\end{abstract}

OCIS codes: (050.5298) Photonic crystals; (130.4815) Optical switching devices.

http://dx.doi.org/10.1364/OL.38.002466

A Fano resonance [1] appears as an interference effect between a discrete level and a continuum in quantum or classical systems. In classical physics, it is observed in systems ranging from waveguide-cavity structures, to plasmonics and metamaterials [2,3]. In waveguide-cavity structures, Fano resonances have been shown both theoretically [ $\underline{4}-\underline{7}]$ and experimentally [ $\underline{8}-10]$ to exhibit sharp asymmetric transmission features. It has been suggested that this increased frequency sensitivity compared to Lorentzian resonances may be useful for applications in switching, electro-optic modulation, and sensing.

In this work, we propose and analyze a simple structure displaying a Fano resonance. It consists of a single photonic crystal cavity coupled to a line-defect waveguide containing a single hole acting as a partially transmitting blockade. The asymmetry of the Fano resonance is characterized by a single parameter (the blockade transmittance $\left|t_{B}\right|$ ), which may be tuned by simply varying the radius of the blockade hole, $r_{b}$. Compared to structures based on an additional cavity $[\underline{4}, \underline{8}]$ or two waveguide blockades [ㅁ, 9$]$, where the asymmetry is characterized by two parameters, our design is simpler to analyze and requires fewer parameters to be controlled accurately in the fabrication process.

We analyze our structure in the context of all-optical switching based on temporal coupled mode theory (TCMT) [11]. The analysis is targeted at investigating the interplay between the sharp transmission features of the Fano resonance and the bandwidth and energy requirements of all-optical switching devices [12]. With our optimized design, we find an order of magnitude reduction in switching energy compared to a structure with a Lorentzian transmission line shape.

Figure 1(a) shows waveguide-cavity structures with different blockade-hole configurations. Light may pass through the structure via coupling to the cavity or directly through the waveguide. A Fano resonance appears if light passing through these paths interferes at the output. The green circles show a conventional design $[\underline{13}, \underline{14}]$ with complete blockade $\left|t_{B}\right|=0$, which results in a
Lorentzian transmission line shape due to the absence of interference. The single red circle shows our design with partial blockade $\left.\left.\left|t_{B}\right| \in\right] 0 ; 1\right]$, and the corresponding transmission spectra calculated from 3D finite-difference time-domain (FDTD) simulations [15] are shown in Fig. 1(b). FDTD modeling is performed using a parallel simulation/optimization package developed in-house [16]. The cavity mode is odd with respect to reflections along the vertical symmetry-plane through the center of the cavity [see Fig. 1(a)]. In this case, the TCMT equations for the signal are [11]

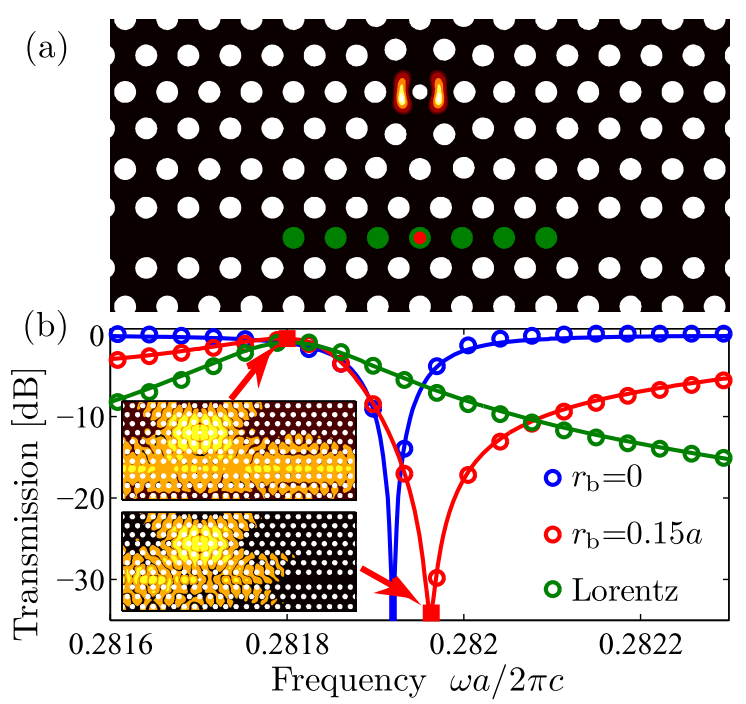

Fig. 1. (a) Photonic crystal waveguide-cavity structure with holes of radii $r_{b}$ in the waveguide (red and green) and $r_{0}$ in the remaining lattice. The field profile $|\mathbf{H}|^{2}$ is overlaid. (b) Transmission spectra found from 3D FDTD calculations (circles) for the different blockade configurations in (a), where $a$ is the lattice constant and $c$ the speed of light. The blue curve corresponds to an unblocked waveguide, and the red and green curves match the colors in (a). The solid lines are fits using Eq. (3). The insets show $|\mathbf{H}|^{2}$ using CW excitation in the "on" and "off" states of the Fano structure as indicated by the red squares. 


$$
\begin{gathered}
\frac{d a_{S}(t)}{d t}=\left(-i\left[\delta_{S}+\delta_{\mathrm{NL}}(t)\right]-\gamma_{C}\right) a_{S}(t)+\sqrt{\gamma} s_{S}^{i}(t), \\
s_{S}^{o}(t)=t_{B} s_{S}^{i}(t)-\sqrt{\gamma} a_{S}(t),
\end{gathered}
$$

where $\left|a_{S}\right|^{2}$ is the energy of the signal inside the cavity, $\left|s_{S}^{i}\right|^{2}\left(\left|s_{S}^{o}\right|^{2}\right)$ is the input (output) power in the waveguide, $\delta_{S}=\omega_{C}-\omega_{S}$ is the detuning, $\gamma_{C}=\Delta \omega_{C} / 2$ is the total decay rate, $\gamma$ is the cavity-waveguide coupling rate, and $\delta_{\mathrm{NL}}$ is the (generally complex) perturbation of the resonance due to nonlinearities. In continuous wave (CW) excitation, the time-derivative of the slowly varying envelope $a_{S}$ equals zero, and the linear transmission spectrum is

$$
T_{\mathrm{CW}}=\left.|| t_{B}\right|^{2}+i\left|t_{B}\right|\left|r_{B}\right|-\left.\frac{\gamma}{i \delta_{S}+\gamma_{C}}\right|^{2},
$$

where $\left|t_{B}\right|^{2}+\left|r_{B}\right|^{2}=1$. From Eq. (3) it is seen that the asymmetry of the transmission is determined by a single parameter (the amplitude of the blockade transmittance). From Fig. 1(b) it is evident that the expression in Eq. (3) accounts very well for the transmission found from FDTD simulations. Also, from this figure, it is clear that for a Fano resonance, a small shift of the resonance can lead to a much larger switching contrast than for the conventional Lorentzian line shape. For high-speed signals, however, it is necessary to consider the finite signal bandwidth and we therefore consider the energy transmission

$$
T_{U}\left(\left|t_{B}\right|, \delta_{S}, \Omega_{S}\right)=\frac{\int T_{\mathrm{CW}}(\omega) P_{S}^{i}\left(\omega, \Omega_{S}\right) \mathrm{d} \omega}{\int P_{S}^{i}\left(\omega, \Omega_{S}\right) \mathrm{d} \omega}
$$

where $P_{S}^{i}$ is the input power and $\Omega_{S}$ is the full-width at half-maximum (FWHM) of the input spectrum.

The signal transmission may be changed dynamically by a (relatively strong) pump pulse, which causes the nonlinear shift $\delta_{\mathrm{NL}}$. To evaluate the switching performance of the structure, some requirements must be imposed on the "on" and "off" state transmission levels $T_{U}^{\mathrm{on}}$ and $T_{U}^{\mathrm{off}}$, corresponding to the pump being present or not. As an example, we require an "on" state transmission $T_{U}^{\mathrm{on}} \geq 0.7$ and a switching contrast $T_{U}^{\mathrm{on}} / T_{U}^{\mathrm{off}} \geq 12 \mathrm{~dB}$ and use Gaussian pulses with FWHM $\Omega_{S / P}$, where the subscripts correspond to the signal and pump, respectively. Under the assumption that the nonlinear effect caused by the pump pulse simply frequency shifts the linear transmission spectrum, Eqs. (3) and (4) contain important information about the maximum achievable switching contrast at any given signal detuning $\delta_{S}$. The maximum "on" state transmission is always largest in the linear regime, because the detuning is constant in time, while it changes dynamically during the passage of the signal in the nonlinear regime. From Eqs. (3) and (4) we can calculate the detuning $\delta_{S}^{\mathrm{min}}$, which will result in a minimum nonlinear shift $\delta_{\mathrm{NL}}^{\min }$, such that the "on" state transmission $T_{U}\left(\delta_{S}^{\min }+\delta_{\mathrm{NL}}^{\min }\right) \geq 0.7$ and the switching contrast $T_{U}\left(\delta_{S}^{\min }+\delta_{\mathrm{NL}}^{\min }\right) / T_{U}\left(\delta_{S}^{\min }\right) \geq 12 \mathrm{~dB}$. This is illustrated in the left panel of Fig. 2 . In the right

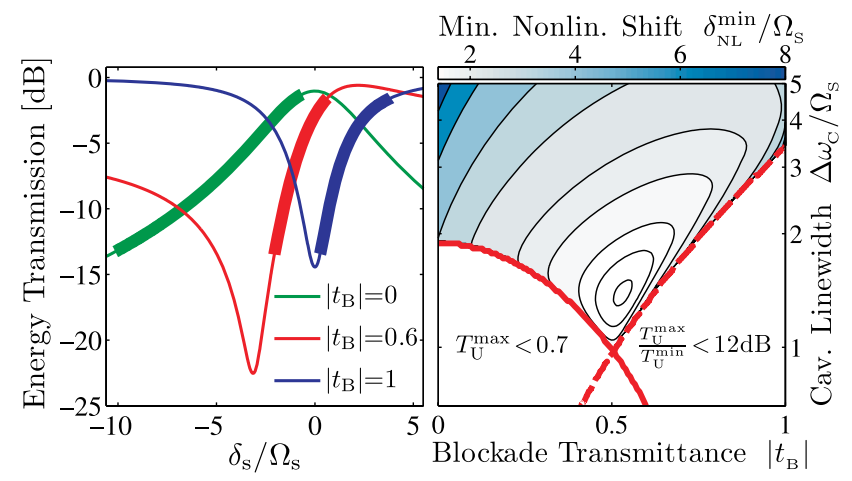

Fig. 2. Left: Energy transmission as a function of detuning for three different values of $\left|t_{B}\right|$ with $\Delta \omega_{C} / \Omega_{S}=5$. The thick parts of the curves indicate the intervals $\left[\delta_{S}^{\min } ; \delta_{S}^{\min }+\delta_{N L}^{\min }\right]$. Right: Minimum nonlinear shift $\delta_{\mathrm{NL}}^{\min }$ as a function of the cavity linewidth $\Delta \omega_{C}$ and blockade transmittance $\left|t_{B}\right|$. The frequency shift and cavity linewidth are normalized with the signal bandwidth $\Omega_{S} . T_{U}^{\max }$ and $T_{U}^{\min }$ are the maximum and minimum transmission for a given parameter set $\left(\left|t_{B}\right|, \Delta \omega_{C}\right)$.

panel we show the dependence of $\delta_{\mathrm{NL}}^{\min }$ on the blockade transmittance and the cavity linewidth for a fixed signal bandwidth. The energy transmission also depends on $\gamma / \gamma_{C}$, and we fix this ratio at $\gamma / \gamma_{C}=0.9$, which is estimated from the FDTD calculations in Fig. 1(b). When the cavity linewidth increases, the transmission broadens, demanding a larger nonlinear shift $\delta_{\mathrm{NL}}^{\mathrm{min}}$. Similarly, cavity linewidths that are small compared to the pulse bandwidth also require a larger nonlinear shift. As a result, there is a minimum in the nonlinear shift for any given blockade transmittance, unless the transmission requirements are violated before this minimum occurs.

The switching energy is defined as the minimum pump energy needed to fulfill the above requirements. The relation between the nonlinear shift $\delta_{\mathrm{NL}}$ and the pump energy inside the cavity depends on the nature of the nonlinearity. The cavity energy, however, increases with decreasing cavity linewidth in a manner that is independent of $\left|t_{B}\right|$, as seen from Eq. (1). Therefore, for any given $\Delta \omega_{C}$, the switching energy is expected to be reduced by choosing $\left|t_{B}\right|$ to minimize $\delta_{\mathrm{NL}}^{\mathrm{min}}$, because it sets a lower bound on the nonlinear shift, and hence, on the required pump energy. From Fig. 2 it is seen that the advantage of the Fano resonance compared to the Lorentzian resonance (at $\left|t_{B}\right|=0$ ) is twofold: First, a smaller nonlinear shift is required, and second, a smaller cavity linewidth may be used without violating the requirements.

As a simple and illustrative example of the connection between the reduction in $\delta_{\mathrm{NL}}^{\mathrm{min}}$ observed in Fig. 2 and the reduction in switching energy, we consider the Kerr effect, where the nonlinear shift is proportional to the pump energy inside the cavity, $\delta_{\mathrm{NL}}(t)=F_{\mathrm{NL}}\left|a_{P}(t)\right|^{2}$. The pump field $a_{P}$ is governed by Eq. (1) with subscripts " $S$ " replaced by " $P$ ". Separation of the equations for $a_{S}$ and $a_{P}$ is possible if we consider a multimode cavity $[12,17]$. For a fixed signal bandwidth $\Omega_{S}$ and $\gamma / \gamma_{C}=$ 0.9 , we vary the blockade transmittance $\left|t_{B}\right|$ and resonance linewidth $\Delta \omega_{C}$. For each parameter set, the detuning is chosen to be $\delta_{S}=\delta_{S}^{\min }$ corresponding to the minimum nonlinear shifts in Fig. 2 . A minimum pump 


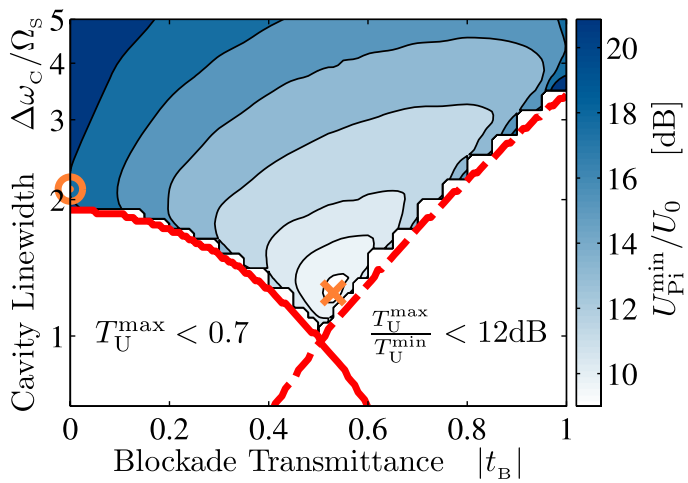

Fig. 3. Minimum switching energy $U_{\mathrm{Pi}}^{\min }$ as a function of the resonance linewidth $\Delta \omega_{C}$ and blockade transmittance $\left|t_{B}\right|$. The characteristic energy is given by $U_{0}=\Omega_{S} / F_{\mathrm{NL}}$. The orange circle indicates the minimum for a Lorentzian line shape, the cross indicates the overall energy minimum, and their ratio of $11 \mathrm{~dB}$ quantifies the achievable energy reduction by using a Fano resonance.

energy $U_{\mathrm{Pi}}^{\min }$ is then found by varying the pump parameters (detuning, bandwidth, and delay with respect to the signal). The result is shown in Fig. 3. The features of Fig. 3 are very similar to those of Fig. 2 . Because $F_{\mathrm{NL}}$ is real in our example, it follows from $\bar{E} q$. (1) that the nonlinear effect only frequency shifts the linear transmission spectrum. Furthermore, $\delta_{\mathrm{NL}}^{\min }$ and $\delta_{\mathrm{NL}}(t)$ scale similarly with the cavity linewidth, because $\delta_{\mathrm{NL}}$ is directly proportional to the cavity energy. In fact, the deviations between Figs. $\underline{3}$ and $\underline{2}$ are caused only by the dynamical shift of the resonance in the nonlinear case, as discussed above. We observe that the optimum switching energy in Fig. $\underline{3}$ corresponding to the Fano resonance with $\left|t_{B}\right| \simeq$ 0.5 is more than an order of magnitude smaller than for the Lorentzian line with $\left|t_{B}\right|=0$. A more elaborate TCMT model is necessary to make predictions about the achievable energy reduction for carrier-induced nonlinearities $[\underline{13}, \underline{14}]$, but from the reduction in $\delta_{\mathrm{NL}}^{\min }$ observed in Fig. 2 , a significant energy reduction is expected in the general case. We notice that if the switched pulses are to be further transmitted, additional limitations on the acceptable spectral distortion may need to be formulated.

In conclusion, we have proposed a simple photonic crystal waveguide-cavity structure that exhibits Fano resonances governed by only a single control parameter that can readily be varied experimentally. Taking into account the bandwidth of the signal and demands for "on" state transmission and switching contrast, we have shown that, in general, a significant energy reduction is expected for a Fano line shape compared to a Lorentzian line shape, with our example showing an order of magnitude reduction. In this work, we focused on the application of Fano resonances in optical switching, but we note that the increased sensitivity to the resonance frequency may find application in a broader range of devices including sensors and modulators.

This work was supported by the Villum Kann Rasmussen Center of Excellence NATEC, the Danish Council for Independent Research (FTP 10-093651), and the EU FP7 project "Copernicus."

\section{References}

1. U. Fano, Phys. Rev. 124, 1866 (1961).

2. A. E. Miroschnichenko, S. Flach, and Y. Kivshar, Rev. Mod. Phys. 82, 2257 (2010).

3. B. Luk'yanchuk, N. I. Zheludev, S. A. Maier, N. J. Halas, P. Nordlander, H. Giessen, and C. T. Chong, Nat. Phys. 9, 707 (2010).

4. S. F. Mingaleev, A. E. Miroschnichenko, and Y. Kivshar, Opt. Express 16, 11647 (2008).

5. S. Fan, App. Phys. Lett. 80, 908 (2002).

6. A. R. Cowan and J. F. Young, Phys. Rev. E 68, 046606 (2003).

7. A. E. Miroschnichenko and Y. Kivshar, Phys. Rev. E 72, 056611 (2005).

8. K. Nozaki, A. Shinya, S. Matsuo, T. Sato, E. Kuramochi, and M. Notomi, Opt. Express 21, 11877 (2013).

9. X. Yang, C. Husko, C. W. Wong, M. Yu, and D. Kwong, App. Phys. Lett. 91, 051113 (2007).

10. K. K. Mehta, J. S. Orcutt, and R. J. Ram, Appl. Phys. Lett. 102, 081109 (2013).

11. S. Fan, W. Suh, and J. D. Joannopoulos, J. Opt. Soc. Am. A 20, 569 (2003)

12. M. Heuck, P. T. Kristensen, and J. Mørk, Opt. Express 19, 18410 (2011).

13. C. Husko, A. de Rossi, S. Combrié, Q. V. Tran, F. Raineri, and C. W. Wong, Appl. Phys. Lett. 94, 021111 (2009).

14. K. Nozaki, T. Tanabe, A. Shinya, S. Matsuo, T. Sato, H. Taniyama, and M. Notomi, Nat. Photonics 4, 477 (2010).

15. A. Taflove and S. C. Hagnes, Computational Electrodynamics-The Finite-Difference Time-Domain Method, 3rd ed. (Artech House, 2005).

16. Y. Elesin, B. S. Lazarov, J. S. Jensen, and O. Sigmund, "Time domain topology optimization of 3D nanotonic devices," Photon. Nanostructures, submitted for publication.

17. Y. Yu, M. Heuck, S. Ek, N. Kuznetsova, K. Yvind, and J. Mørk, Appl. Phys. Lett. 101, 25 (2012). 